\title{
Leptonic and semileptonic $D$ meson decays
}

\author{
Hailong Ma (For BESIII Collaboration) ${ }^{* \dagger}$ \\ Institute of High Energy Physics, Chinese Academy of Sciences \\ E-mail: mahleihep.ac.cn
}

Herein, we report recently improved measurements of the decay constants $f_{D_{(s)}^{+}}$, the form factors $f_{+}^{K(\pi)}(0)$, and the CKM matrix element $\left|V_{c s(d)}\right|$, which are made based on analysis of $D_{(s)}^{+} \rightarrow \ell^{+} v_{\ell}$ and $D^{0} \rightarrow K(\pi)^{-} e^{+} v_{e}$ at BABAR, BELLE and BESIII. These provide important information to better validate the LQCD calculations of $f_{D_{(s)}^{+}}$and $f_{+}^{K(\pi)}(0)$, and to better test the unitarity of the CKM matrix.

The 15th International Conference on B-Physics at Frontier Machines at the University of Edinburgh, 14 -18 July, 2014

University of Edinburgh, $U K$

\footnotetext{
* Speaker.

$\dagger$ I would like to thank for the support of the National Natural Science Foundation of China (NSFC) under Contracts No. 11305180 and No. 10935007, and the Ministry of Science and Technology of China (973 by MOST) under Contract of 2009CB825200.
} 


\section{Introduction}

In the Standard Model, the $D_{(s)}^{+}$mesons decay into $\ell v_{\ell}$ via a virtual $W^{+}$boson. The decay rate of the leptonic decays $D_{(s)}^{+} \rightarrow \ell^{+} v_{\ell}$ can be parameterized by the $D_{(s)}^{+}$decay constant $f_{D_{(s)}^{+}}$via

$$
\Gamma\left(D_{(s)}^{+} \rightarrow \ell^{+} v_{\ell}\right)=\frac{G_{F}^{2}}{8 \pi}\left|V_{\mathrm{cd}(\mathrm{s})}\right|^{2} f_{D_{(s)}^{+}} m_{\ell}^{2} m_{D_{(s)}^{+}}\left(1-\frac{m_{\ell}^{2}}{m_{D_{(s)}^{+}}^{2}}\right),
$$

where $G_{F}$ is the Fermi coupling constant, $\left|V_{\mathrm{cd}(\mathrm{s})}\right|$ is the CKM matrix element between the two quarks $c \bar{d}(\bar{s}), m_{\ell}$ and $m_{D_{(s)}^{+}}$are the masses of the lepton and $D_{(s)}^{+}$.

On the other hand, the semileptonic decays $D^{0} \rightarrow K(\pi)^{-} e^{+} v_{e}$ can be well interpreted because the effects of the weak and strong interaction can be well separated in theory. Their differential decay rates can be simply written as

$$
\frac{d \Gamma}{d q^{2}}=\frac{G_{F}^{2}}{24 \pi^{3}}\left|V_{\mathrm{cs}(\mathrm{d})}\right|^{2} p_{K(\pi)}^{3}\left|f_{+}^{K(\pi)}\left(q^{2}\right)\right|^{2},
$$

where $G_{F}$ is the Fermi coupling constant, $\left|V_{\mathrm{cs}(\mathrm{d})}\right|$ is the CKM matrix element between the two quarks $c \bar{s}(\bar{d}), p_{K(\pi)}$ is the momentum of the kaon(pion) in the rest frame of $D^{0}$ meson, $f_{+}^{K(\pi)}\left(q^{2}\right)$ is the form factor of hadronic weak current depending on the square of the four momentum transfer $q=p_{D}-p_{K(\pi)}$.

The world largest $e^{+} e^{-}$annihilation data set taken at $\psi(3770)$ with the BESIII detector, as well as the huge $e^{+} e^{-}$annihilation data sets taken at $\Upsilon(4 S)$ with the BABAR and BELLE detectors provide good opportunities to better study the leptonic decays $D_{(s)}^{+} \rightarrow \ell^{+} v_{\ell}$ and the semileptonic decays $D^{0} \rightarrow K(\pi)^{-} e^{+} v_{e}$. Based on these, the decay constants of $D_{(s)}^{+}$, the form factors $f_{+}^{K(\pi)}\left(q^{2}\right)$ or the CKM matrix elements $\left|V_{\mathrm{cd}(\mathrm{s})}\right|$ can be accurately extracted, thus precisely validating the LQCD calculations on $f_{D_{(s)}^{+}}$and $f_{+}^{K(\pi)}(0)$ and/or precisely testing the unitarity of the CKM matrix. Also, these are helpful to improve the measurement accuracies in the experimental studies of the leptonic and semileptonic decays of B mesons indirectly.

Herein, some recent results on the leptonic decay $D^{+} \rightarrow \mu^{+} v_{\mu}$ and the semileptonic decays $D^{0} \rightarrow K(\pi)^{-} e^{+} v_{e}$ at the BESIII, as well as the leptonic decay $D_{s}^{+} \rightarrow \ell^{+} v_{\ell}(\ell=\mu, \tau)$ and the semileptonic decays $D^{0} \rightarrow K(\pi)^{-} e^{+} v_{e}$ at the BABAR and BELLE are reported. Throughout the proceeding, the charge conjugate is implied.

\section{Leptonic decays $D_{(s)}^{+} \rightarrow \ell^{+} v_{\ell}$}

\section{1 $D^{+} \rightarrow \mu^{+} v_{\mu}$ at the BESIII [3]}

In 2010 and 2011, the BESIII [1] accumulated $2.92 \mathrm{fb}^{-1}$ data at $\sqrt{s}=3.773 \mathrm{GeV}$ [2], where $e^{+} e^{-} \rightarrow \psi(3770) \rightarrow D^{0} \bar{D}^{0}\left(D^{+} D^{-}\right)$is produced predominantly, thus providing ideal place to study the decays of $D^{0}$ and $D^{+}$mesons. To investigate $D^{+} \rightarrow \mu^{+} v_{\mu}$, BESIII reconstructs singly tagged $D^{-}$mesons by using 9 hadronic decays. Figure 1 (left side) shows the fits to the beam-energyconstrained mass spectra of the (a) $K^{+} \pi^{-} \pi^{-}$, (b) $K_{S}^{0} \pi^{-}$, (c) $K_{S}^{0} K^{-}$, (d) $K^{+} K^{-} \pi^{-}$, (e) $K^{+} \pi^{-} \pi^{-} \pi^{0}$, (f) $\pi^{-} \pi^{-} \pi^{+}$, (g) $K_{S}^{0} \pi^{-} \pi^{0}$, (h) $K^{+} \pi^{-} \pi^{-} \pi^{-} \pi^{+}$and (i) $K_{S}^{0} \pi^{-} \pi^{-} \pi^{+}$combinations, which yield $(170.31 \pm 0.34) \times 10^{4}$ singly tagged $D^{-}$mesons.

Figure 1 (right side) shows the $M_{\text {miss }}^{2}$ distribution of the candidates for $D^{+} \rightarrow \mu^{+} v_{\mu}$, which are selected in the systems against the singly tagged $D^{-}$mesons. BESIII obtains $409 \pm 21$ signal events of $D^{+} \rightarrow \mu^{+} v_{\mu}$, which yields the branching fraction for $D^{+} \rightarrow \mu^{+} v_{\mu}$

$$
B\left(D^{+} \rightarrow \mu^{+} v_{\mu}\right)=\left(3.71 \pm 0.19_{\text {stat. }} \pm 0.06_{\text {sys. }}\right) \times 10^{-4} .
$$



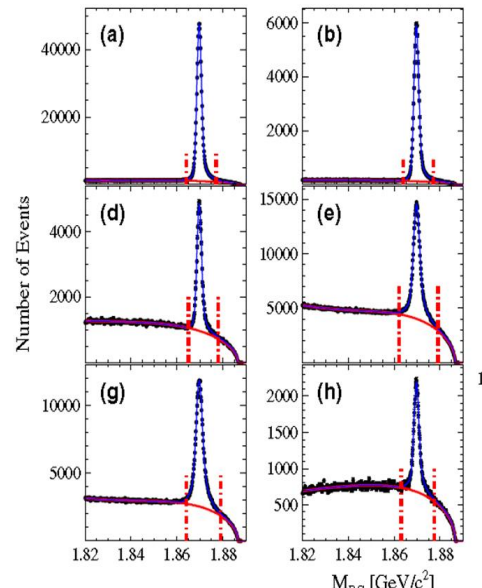

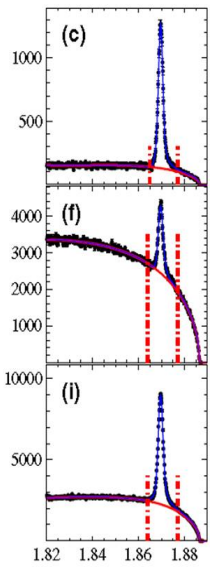

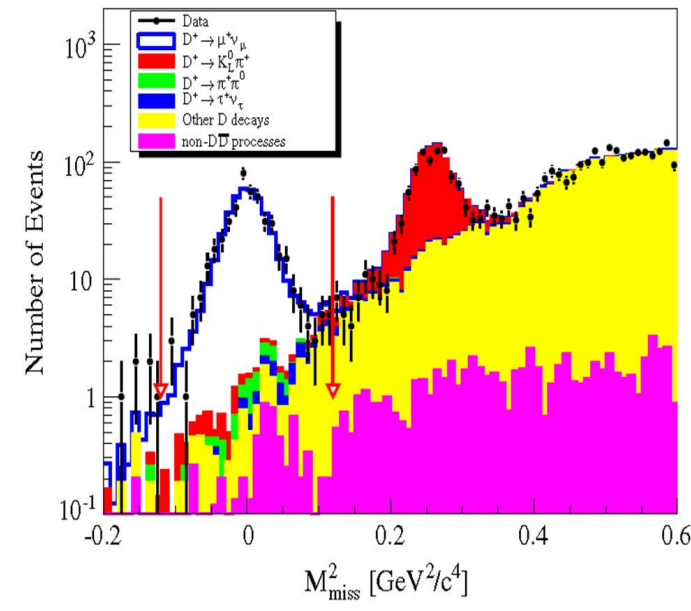

Figure 1: (left side) The fits to the beam-energy-constrained mass spectra for singly tagged $D^{-}$modes (the signal region is marked by the pair of arrows in each sub-figure). (right side) The $M_{\text {miss }}^{2}$ distribution for $D^{+} \rightarrow \mu^{+} v_{\mu}$ candidates.

Using the measured $B\left(D^{+} \rightarrow \mu^{+} v_{\mu}\right)$ and the CKM matrix element $\left|V_{\mathrm{cd}}\right|$ value determined from a global Standard Model fit [4], BESIII determines the decay constant of $D^{+}$to be

$$
f_{D^{+}}=203.2 \pm 5.3_{\text {stat. }} \pm 1.8_{\text {sys. }} \mathrm{MeV} .
$$

The $B\left(D^{+} \rightarrow \mu^{+} v_{\mu}\right)$ and $f_{D^{+}}$measured at BESIII are consistent within errors with those measured at the BESI [5], BESII [6] and CLEO-c [7] experiments, but with the best precision.

So far, the CKM matrix element $\left|V_{\mathrm{cd}}\right|$ has been measured though experimental studies of the semileptonic decay $D \rightarrow \pi \ell^{+} v_{\ell}$ or measurement of charm production cross section of $v \bar{v}$ interaction, among which the best measurement precision is 4.8\% [4]. By using the measured $B\left(D^{+} \rightarrow \mu^{+} v_{\mu}\right)$ and the Lattice QCD calculation on $f_{D^{+}}$[8], BESIII determines the CKM matrix element $\left|V_{\text {cd }}\right|$ to be

$$
\left|V_{\text {cd }}\right|=0.2210 \pm 0.058_{\text {stat. }} \pm 0.047_{\text {sys. }},
$$

which has the best precision in the world to date.

\section{2 $D_{s}^{+} \rightarrow \ell^{+} v_{\ell}$ at the BABAR and BELLE}

In 2010, the BABAR Collaboration investigated $D_{s}^{+} \rightarrow \ell^{+} v_{\ell}$ by analyzing $521 \mathrm{fb}^{-1}$ data taken at $\sqrt{s}=10.58 \mathrm{GeV}$ [9]. They accumulated $(67.2 \pm 1.5) \times 10^{3}$ inclusive $D_{s}^{+}$mesons by the decay chain $e^{+} e^{-} \rightarrow c \bar{c} \rightarrow D K X D_{s}^{*-}$. From the inclusive $D_{s}^{+}$sample, they obtained $275 \pm 17,408 \pm 42$ and $340 \pm 32$ signals for $D_{s}^{+} \rightarrow \mu^{+} v_{\mu}, D_{s}^{+} \rightarrow \tau^{+} v_{\tau}$ with $\tau^{+} \rightarrow e^{+} v \bar{v}$ and $D_{s}^{+} \rightarrow \tau^{+} v_{\tau}$ with $\tau^{+} \rightarrow \mu^{+} \nu \bar{v}$. Based on these, they measured the branching fraction for $D_{s}^{+} \rightarrow \ell^{+} v_{\ell}$ to be

$$
B\left(D_{s}^{+} \rightarrow \mu^{+} v_{\mu}\right)=\left(0.602 \pm 0.038_{\text {stat. }} \pm 0.034_{\text {sys. }}\right) \%
$$

and

$$
B\left(D_{s}^{+} \rightarrow \tau^{+} v_{\tau}\right)=\left(5.00 \pm 0.35_{\text {stat. }} \pm 0.49_{\text {sys. }}\right) \% .
$$

Further, they determined the decay constant of $D_{s}^{+}$to be

$$
f_{D_{s}^{+}}=258.6 \pm 6.4_{\text {stat. }} \pm 7.5_{\text {sys. }} \mathrm{MeV} .
$$

In 2013, the BELLE Collaboration investigated $D_{s}^{+} \rightarrow \ell^{+} v_{\ell}$ by analyzing $913 \mathrm{fb}^{-1}$ data taken at $\sqrt{s}=10.58 \mathrm{GeV}[10]$. They accumulated $94360 \pm 1310_{\text {stat. }} \pm 1450_{\text {sys. }}$ inclusive $D_{s}^{+}$mesons 

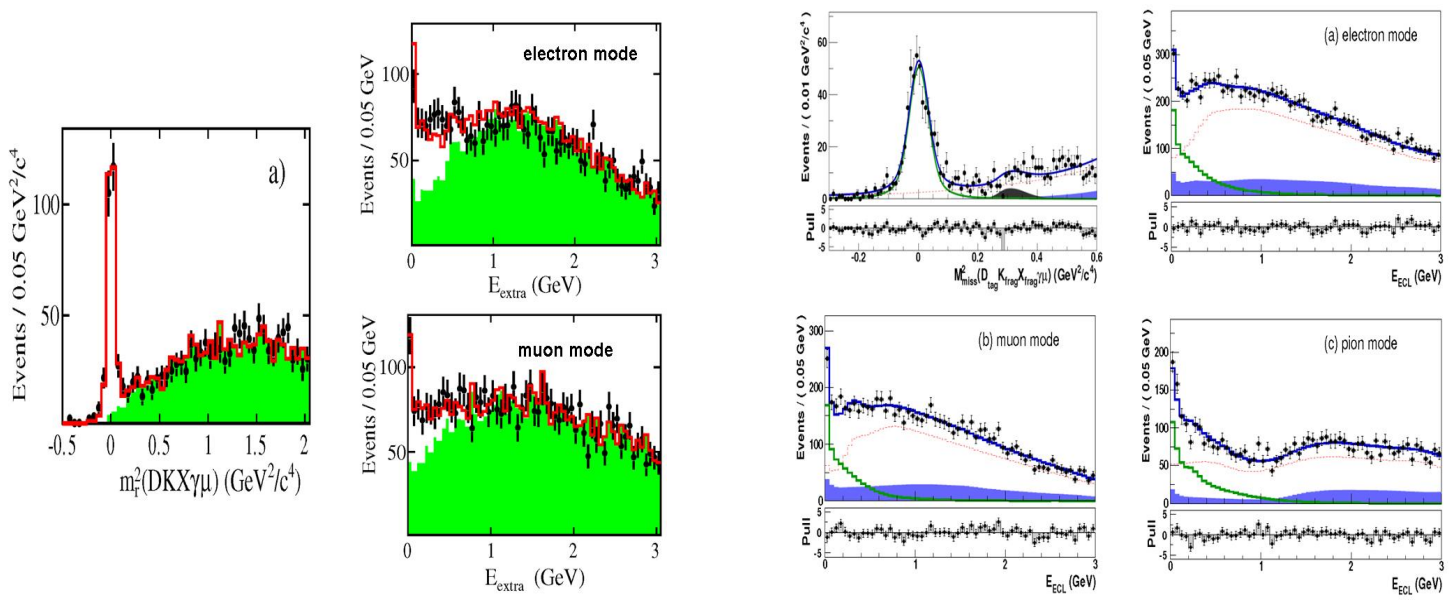

Figure 2: The distributions of the missing mass squares of the candidates for $D_{s}^{+} \rightarrow \mu^{+} v_{\mu}$ and the extra energies of the candidates for $D_{s}^{+} \rightarrow \ell^{+} v_{\ell}$ at the BABAR (left side) and the BELLE (right side).

by the decay chain $e^{+} e^{-} \rightarrow c \bar{c} \rightarrow D K X D_{s}^{*-}$. From the inclusive $D_{s}^{+}$sample, they found $492 \pm 26$, $952 \pm 59,758 \pm 48$ and $496 \pm 35$ signals for $D_{s}^{+} \rightarrow \mu^{+} v_{\mu}, D_{s}^{+} \rightarrow \tau^{+} v_{\tau}$ with $\tau^{+} \rightarrow e^{+} v \bar{v}, D_{s}^{+} \rightarrow \tau^{+} v_{\tau}$ with $\tau^{+} \rightarrow \mu^{+} \nu \bar{v}$ and $D_{s}^{+} \rightarrow \tau^{+} v_{\tau}$ with $\tau^{+} \rightarrow \pi^{+} v$. Based on these, they measured the branching fraction for $D_{s}^{+} \rightarrow \ell^{+} v_{\ell}$ to be

$$
B\left(D_{s}^{+} \rightarrow \mu^{+} v_{\mu}\right)=\left(0.531 \pm 0.028_{\text {stat. }} \pm 0.020_{\text {sys. }}\right) \%
$$

and

$$
B\left(D_{s}^{+} \rightarrow \tau^{+} v_{\tau}\right)=\left(5.70 \pm 0.21_{\text {stat. }} \pm 0.31_{\text {sys. }}\right) \% .
$$

Further, they determined the decay constant of $D_{s}^{+}$to be

$$
f_{D_{s}^{+}}=255.5 \pm 4.2_{\text {stat. }} \pm 5.1_{\text {sys. }} \mathrm{MeV} \text {. }
$$

\subsection{Comparison of the extracted $f_{D_{(s)}^{+}}$}

Figure 3 compares the measured $f_{D^{+}}$at the BESIII and the measured $f_{D_{s}^{+}}$at the BABAR and BELLE with the ones from other experiments or theory calculation.

\section{Semileptonic decays $D^{0} \rightarrow K(\pi)^{-} e^{+} v_{e}$}

\subsection{Previous or preliminary results at the BABAR and BELLE}

In 2006, the BELLE Collaboration investigated $D^{0} \rightarrow K(\pi)^{-} e^{+} v_{e}$ by analyzing $282 \mathrm{fb}^{-1}$ data taken at $\sqrt{s}=10.58 \mathrm{GeV}$ [11]. They determined the branching fractions for $D^{0} \rightarrow K(\pi)^{-} e^{+} v_{e}$ which are $B\left(D^{0} \rightarrow K^{-} e^{+} v_{e}\right)=\left(3.45 \pm 0.10_{\text {stat. }} \pm 0.19_{\text {sys. }}\right) \%$ and $B\left(D^{0} \rightarrow \pi^{-} e^{+} v_{e}\right)=(0.279 \pm$ $\left.0.027_{\text {stat. }} \pm 0.016_{\text {sys. }}\right) \%$. By fitting the decay widths for $D^{0} \rightarrow K(\pi)^{-} \ell^{+} v_{\ell}(\ell=e, \mu)$, they extracted the form factors $f_{+}^{K}(0)=0.695 \pm 0.007 \pm 0.022$ and $f_{+}^{\pi}(0)=0.624 \pm 0.020 \pm 0.030$.

In 2007, by analyzing $75 \mathrm{fb}^{-1}$ data taken at $\sqrt{s}=10.58 \mathrm{GeV}$, the BABAR Collaboration studied $D^{0} \rightarrow K^{-} e^{+} v_{e}$ [12]. Using a similar analysis, they determined the branching fraction to be $B\left(D^{0} \rightarrow K^{-} e^{+} v_{e}\right)=\left(3.522 \pm 0.027_{\text {stat. }} \pm 0.079_{\text {sys. }}\right) \%$, and extracted the form factor to be $f_{+}^{K}(0)=$ $0.727 \pm 0.007 \pm 0.086$. At ICHEP2014, the BABAR Collaboration reported preliminary results on $D^{0} \rightarrow \pi^{-} e^{+} v_{e}$ based on a similar analysis of $347.2 \mathrm{fb}^{-1}$ data taken at $\sqrt{s}=10.58 \mathrm{GeV}$ [13]. They determined $B\left(D^{0} \rightarrow \pi^{-} e^{+} v_{e}\right)=\left(2.770 \pm 0.068_{\text {stat. }} \pm 0.099_{\text {sys. }}\right) \times 10^{-3}$, and extracted the form factor to be $f_{+}^{\pi}(0)=0.610 \pm 0.017 \pm 0.011$ or the CKM matrix element $\left|V_{\mathrm{cd}}\right|$ to be $\left|V_{\mathrm{cd}}\right|=$ $0.206 \pm 0.007 \pm 0.009_{\mathrm{LQCD}}$. 

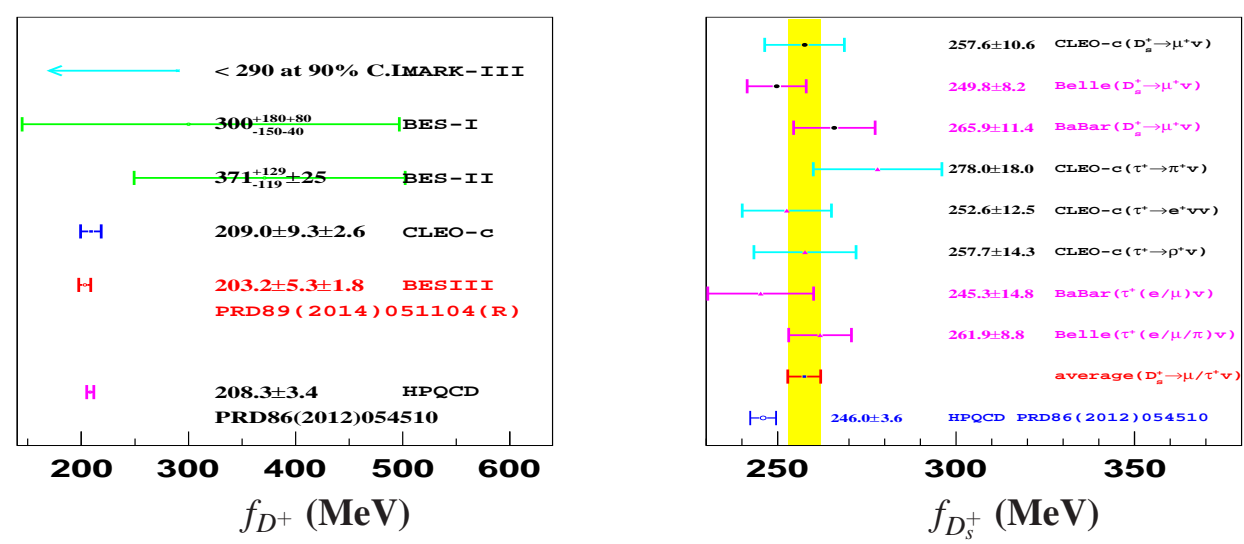

Figure 3: Comparison of the extracted decay constants.

\subsection{Preliminary results at the BESIII}

To investigate $D^{0} \rightarrow K(\pi)^{-} e^{+} v_{e}$, BESIII reconstructs singly tagged $\bar{D}^{0}$ mesons by using 5 hadronic decays. Figure 4 (left side) shows the fits to the beam-energy-constrained mass spectra of (a) $K^{+} \pi^{-}$, (b) $K^{+} \pi^{-} \pi^{0}$, (c) $K^{+} \pi^{-} \pi^{-} \pi^{+}$, (d) $K^{+} \pi^{-} \pi^{-} \pi^{+} \pi^{0}$ and (e) $K^{+} \pi^{-} \pi^{0} \pi^{0}$ combinations from $2.92 \mathrm{fb}^{-1}$ data taken at $\sqrt{s}=3.773 \mathrm{GeV}$. From the fits, BESIII obtains $(279.33 \pm 0.37) \times 10^{4}$ singly tagged $\bar{D}^{0}$ mesons in the $\bar{D}^{0}$ signal region.
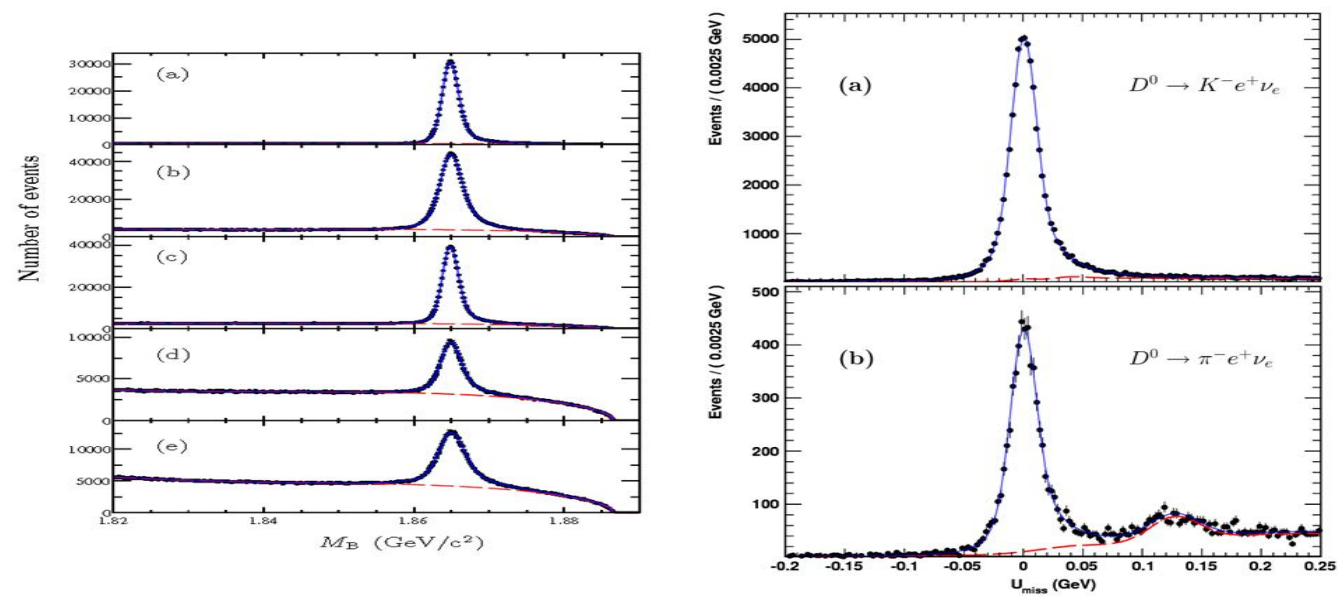

Figure 4: (left side) The fits to the beam-energy-constrained mass spectra for singly tagged $\bar{D}^{0}$ modes. (right side) The $U_{\text {miss }}$ fits for $D^{0} \rightarrow K^{-} e^{+} v_{e}$ and $D^{0} \rightarrow \pi^{-} e^{+} v_{e}$ candidates.

Figure 4 (right side) shows the fits to the $U_{\text {miss }}$ distributions of the candidates for $D^{0} \rightarrow K^{-} e^{+} v_{e}$ and $D^{0} \rightarrow \pi^{-} e^{+} v_{e}$, which are selected in the events containing singly tagged $\bar{D}^{0}$ mesons. From the fits, BESIII obtains $70727 \pm 278$ and $6297 \pm 87$ signal events of $D^{0} \rightarrow K^{-} e^{+} v_{e}$ and $D^{0} \rightarrow \pi^{-} e^{+} v_{e}$. Based on these, the branching fractions for $D^{0} \rightarrow K^{-} e^{+} v_{e}$ and $D^{0} \rightarrow \pi^{-} e^{+} v_{e}$ are determined to be

$$
B\left(D^{0} \rightarrow K^{-} e^{+} v_{e}\right)=\left(3.505 \pm 0.014_{\text {stat. }} \pm 0.033_{\text {sys. }}\right) \%
$$

and

$$
B\left(D^{0} \rightarrow \pi^{-} e^{+} v_{e}\right)=\left(0.2950 \pm 0.0041_{\text {stat. }} \pm 0.0026_{\text {sys. }}\right) \%
$$


respectively. The measured $B\left(D^{0} \rightarrow K^{-} e^{+} v_{e}\right)$ and $B\left(D^{0} \rightarrow \pi^{-} e^{+} v_{e}\right)$ at the BESIII are consistent within errors with those measured at the BESII [14], CLEO-c [15], BELLE [11] and BABAR [12, 13] experiments, but with the best precision.

Figure 5 shows the fits to the partial widths and the projections on the form factors of $D^{0} \rightarrow$ $K^{-} e^{+} v_{e}$ and $D^{0} \rightarrow \pi^{-} e^{+} v_{e}$ using the Simple Pole model [16], the Modified Pole model [16], the ISGW2 model [17], the two-parameter series expansion (Series.2.Par.) [18] and the threeparameter series expansion (Series.3.Par.) [18]. From the fits, BESIII obtains the extracted parameters of different models, which are summarized in Table 1 .
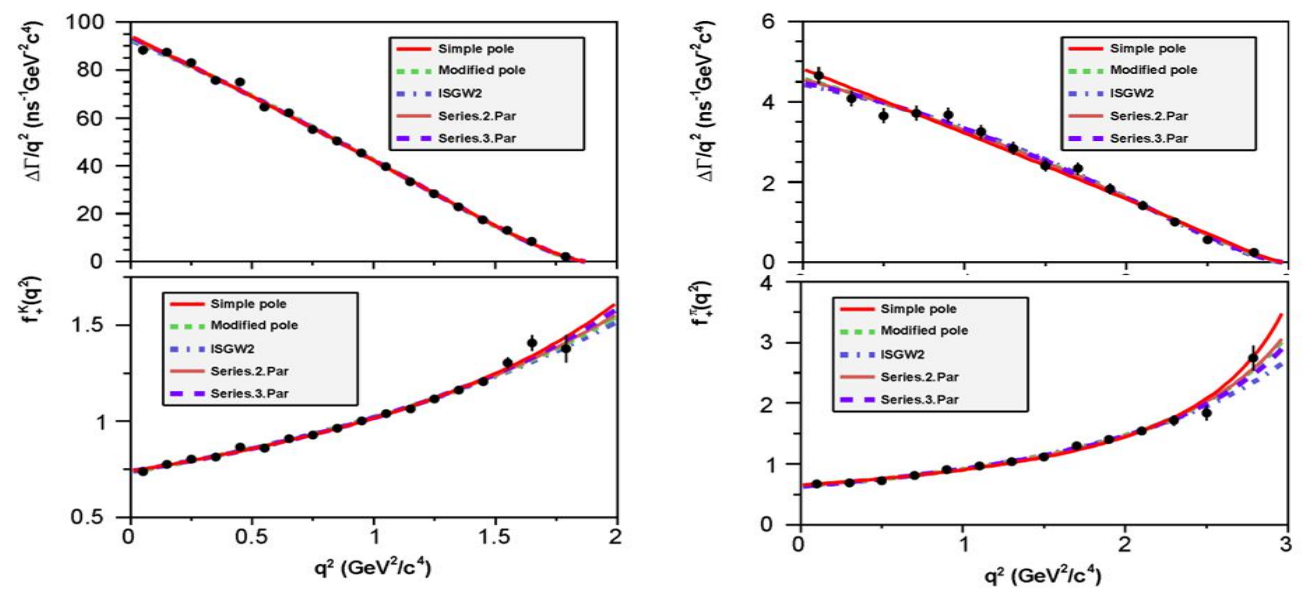

Figure 5: The fits to the partial widths and the projections on the form factors of $D^{0} \rightarrow K^{-} e^{+} v_{e}$ (left side) and $D^{0} \rightarrow \pi^{-} e^{+} v_{e}$ (right side).

Table 1: Summary of the extracted parameters from the fits to the partial widths.

\begin{tabular}{|l|c|c|c|c|}
\hline Model & \multicolumn{2}{|c|}{$D^{0} \rightarrow K^{-} e^{+} v_{e}$} & \multicolumn{2}{c|}{$D^{0} \rightarrow \pi^{-} e^{+} v_{e}$} \\
\hline Simple Pole & $f_{+}^{K}(0)\left|V_{\mathrm{cs}}\right|$ & $0.7209 \pm 0.0022 \pm 0.0033$ & $f_{+}^{\pi}(0)\left|V_{\mathrm{cs}}\right|$ & $0.1475 \pm 0.0014 \pm 0.0005$ \\
& $M_{\mathrm{pole}}$ & $1.9207 \pm 0.0103 \pm 0.0069$ & $M_{\mathrm{pole}}$ & $1.9114 \pm 0.0118 \pm 0.0038$ \\
\hline Modified Pole & $f_{+}^{K}(0)\left|V_{\mathrm{cs}}\right|$ & $0.7163 \pm 0.0024 \pm 0.0034$ & $f_{+}^{\pi}(0)\left|V_{\mathrm{cs}}\right|$ & $0.1437 \pm 0.0017 \pm 0.0008$ \\
& $\alpha$ & $0.3088 \pm 0.0195 \pm 0.0129$ & $\alpha$ & $0.2794 \pm 0.0345 \pm 0.0113$ \\
\hline ISGW2 & $f_{+}^{K}(0)\left|V_{\mathrm{cs}}\right|$ & $0.7139 \pm 0.0023 \pm 0.0034$ & $f_{+}^{\pi}(0)\left|V_{\mathrm{cs}}\right|$ & $0.1415 \pm 0.0016 \pm 0.0006$ \\
& $r_{S G G W 2}$ & $1.6000 \pm 0.0141 \pm 0.0091$ & $r_{S G G 2}$ & $2.0688 \pm 0.0394 \pm 0.0124$ \\
\hline Series.2.Par. & $f_{+}^{K}(0)\left|V_{\mathrm{cs}}\right|$ & $0.7172 \pm 0.0025 \pm 0.0035$ & $f_{+}^{\pi}(0)\left|V_{\mathrm{cs}}\right|$ & $0.1435 \pm 0.0018 \pm 0.0009$ \\
& $r_{1}$ & $-2.2278 \pm 0.0864 \pm 0.0575$ & $r_{1}$ & $-2.0365 \pm 0.0807 \pm 0.0260$ \\
\hline Series.3.Par. & $f_{+}^{K}(0)\left|V_{\mathrm{cs}}\right|$ & $0.7196 \pm 0.0035 \pm 0.0041$ & $f_{+}^{\pi}(0)\left|V_{\mathrm{cs}}\right|$ & $0.1420 \pm 0.0024 \pm 0.0010$ \\
& $r_{1}$ & $-2.3331 \pm 0.1587 \pm 0.0804$ & $r_{1}$ & $-1.8434 \pm 0.2212 \pm 0.0690$ \\
& $r_{2}$ & $3.4223 \pm 3.9090 \pm 2.4092$ & $r_{2}$ & $-1.3871 \pm 1.4615 \pm 0.4677$ \\
\hline
\end{tabular}

\subsection{Comparison of $\left|f_{+}^{K(\pi)}(0)\right|$}

With the measured $f_{+}^{K(\pi)}(0)\left|V_{\mathrm{cs}(\mathrm{d})}\right|$ and the CKM matrix element $\left|V_{\mathrm{cs}(\mathrm{d})}\right|$ values determined from a global Standard Model fit [4], one can calculate the form factors $\left|f_{+}^{K(\pi)}(0)\right|$. Figure 6 compares the calculated form factors $\left|f_{+}^{K(\pi)}(0)\right|$ based on the preliminary results of $f_{+}^{K(\pi)}(0)\left|V_{\mathrm{cs}(\mathrm{d})}\right|$ at BESIII with the ones from other experiments or theory calculation. 

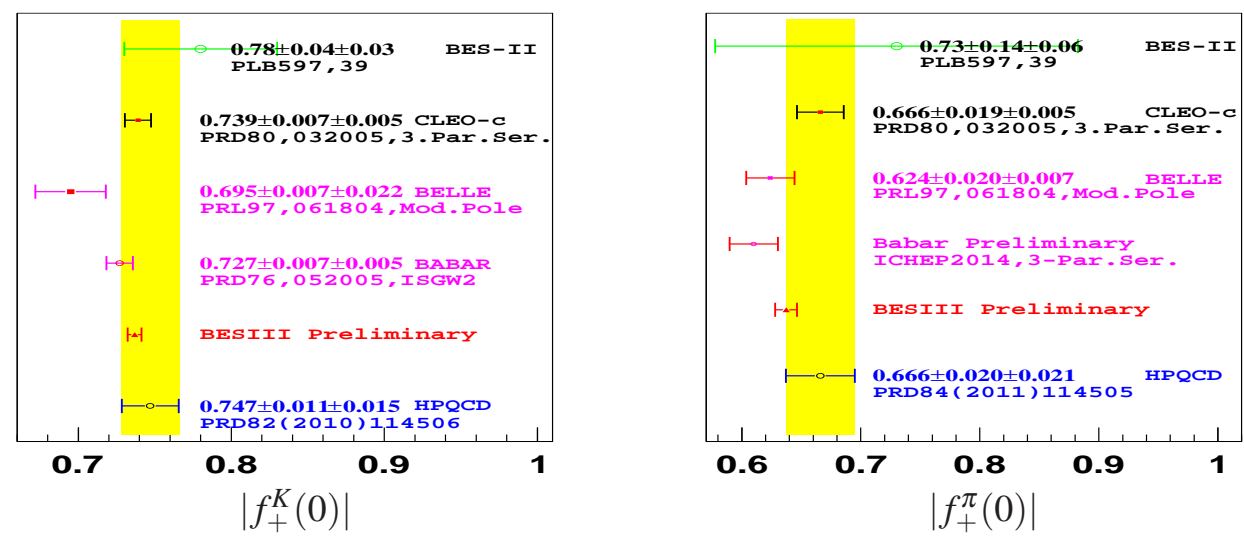

Figure 6: Comparison of the extracted form factors.

\section{Summary}

During the past several years, precision measurements on $D_{(s)}^{+} \rightarrow \ell^{+} v_{\ell}$ and $D^{0} \rightarrow K(\pi)^{-} e^{+} v_{e}$ had been made by the CLEO-c, BABAR, BELLE and BESIII. These improved measurements provide important information to better validate the LQCD calculations of the decay constants $f_{D_{(s)}^{+}}$ and the form factors $f_{+}^{K(\pi)}(0)$, and to better test the unitarity of the CKM matrix. In the near future, BESIII plans to collect $3 \mathrm{fb}^{-1}$ data at $\sqrt{s}=4.17 \mathrm{GeV}$. Also, more data are expected to be collected at $\sqrt{s}=3.773 \mathrm{GeV}$. These will provide new chances to further improve the measurements based on the leptonic decays $D_{(s)}^{+} \rightarrow \ell^{+} v_{\ell}$ and the semileptonic decays $D^{0} \rightarrow K(\pi)^{-} e^{+} v_{e}$.

\section{References}

[1] BESIII Collaboration, M. Ablikim, et al., Nucl. Phys. Method A 614, 345 (2010).

[2] BESIII Collaboration, M. Ablikim, et al., Chinese Physics C 37, 123001 (2013).

[3] BESIII Collaboration, M. Ablikim, et al., Phys. Rev. D 89, 051104(R) (2014).

[4] Particle Data Group, K.A. Olive et al., Chinese Phys. C 38, 090001 (2014).

[5] BES Collaboration, J. Z. Bai, et al., Phys. Lett. B 429, 188 (1998).

[6] BES Collaboration, M. Ablikim, et al., Phys. Lett. B 610, 173 (2005).

[7] CLEO Collaboration, B. I. Eisenstein et al., Phys. Rev. D 78, 052003 (2008).

[8] HPQCD and UKQCD Collaborations, E. Follana et al., Phys. Rev. Lett. 100, 062002 (2008).

[9] BABAR Collaboration, P. del Amo Sanchez et al., Phys. Rev. D 82, 091103(R) (2010).

[10] BELLE Collaboration, A. Zupanc et al., JHEP1309, 129 (2013).

[11] BELLE Collaboration, L. Widhalm, et al., Phys. Rev. Lett. 97, 061804 (2006).

[12] BABAR Collaboration, B. Aubert et al., Phys. Rev. D 76, 052005 (2007).

[13] Arantza Oyanguren (For BABAR Collaboration), presented at ICHEP2014.

[14] BES Collaboration, M. Ablikim, et al., Phys. Lett. B 597, 39 (2005).

[15] CLEO Collaboration, D. Besson, et al., Phys. Rev. D 80, 032005 (2009).

[16] D. Becirevic and A.B. Kaidalov, Phys. Lett. B 478, 417 (2000).

[17] D. Scora and N. Isgur, Phys. Rev. D 52, 2783 (1995).

[18] Thomas Becher, Richard J. Hill, Phys. Lett. B 633, 61 (2006). 